\section{RMD Open}

Rheumatic \& Musculoskeletal Diseases

\title{
Cartilage-gut-microbiome axis: a new paradigm for novel therapeutic opportunities in osteoarthritis
}

To cite: Berthelot J-M, Sellam J, Maugars Y, et al. Cartilage-gut-microbiome axis: a new paradigm for novel therapeutic opportunities in osteoarthritis. RMD Open 2019:5:e001037. doi:10.1136/ rmdopen-2019-001037

Received 17 June 2019 Revised 31 July 2019 Accepted 6 September 2019

Check for updates

(c) Author(s) (or their employer(s)) 2019. Re-use permitted under CC BY-NC. No commercial re-use. See rights and permissions. Published by BMJ.

${ }^{1}$ Rheumatology Unit, Nantes University Hospital, CHU Nantes, 44093 Nantes Cedex 01, France ${ }^{2}$ Sorbonne University, Paris, France

${ }^{3}$ INSERM UMRS_938, CRSA,

Paris, France

${ }^{4}$ Department of Rheumatology, Assistance Publique - Hôpitaux de Paris (AP-HP), Saint- Antoine Hospital, DMU 3iD, Paris, France

Correspondence to Dr Jean-Marie Berthelot; jeanmarie.berthelot@chunantes.fr

\section{ABSTRACT}

DNA of gut microbiota can be found in synovium of osteoarthritis and rheumatoid arthritis. This finding could result from the translocation of still alive bacteria from gut to joints through blood, since the diversified dormant microbiota of healthy human blood can be transiently resuscitated in vitro. The recent finding of gut microbiome in human cartilage, which differed between osteoarthritis and controls, suggests that a similar trafficking of dead or alive bacteria from gut microbiota physiologically occurs between gut and epiphysial bone marrow. Subchondral microbiota could enhance cartilage healing and transform components of deep cartilage matrix in metabolites with immunosuppressive properties. The differences of microbiome observed between hip and knee cartilage, either in osteoarthritis or controls, might be the counterpart of subtle differences in chondrocyte metabolism, themselves in line with differences in DNA methylation according to joints. Although bacteria theoretically cannot reach chondrocytes from the surface of intact cartilage, some bacteria enter the vascular channels of the epiphysial growth cartilage in young animals, whereas others can infect chondrocytes in vitro. In osteoarthritis, the early osteochondral plate angiogenesis may further enhance the ability of microbiota to locate close to the deeper layers of cartilage, and this might lead to focal dysbiosis, low-grade inflammation, cartilage degradation, epigenetic changes in chondrocytes and worsening of osteoarthritis. More studies on cartilage across different ethnic groups, weights, and according to age, are needed, to confirm the silent presence of gut microbiota close to human cartilage and better understand its physiologic and pathogenic significance.

\section{MICROBIOTA CONTRIBUTES TO OSTEOARTHRITIS PATHOGENESIS}

Osteoarthritis (OA) may be an evolutionary mismatch disease, more common today than in the past, because genes inherited from previous generations are inadequately or imperfectly adapted to modern environmental conditions, including physical inactivity and dietary changes leading to obesity and metabolic syndrome. ${ }^{1}$ Interestingly, all those conditions are associated with changes
Key messages

What does this review add?

- DNA of gut microbiome could be found in synovium of osteoarthritis but also in human cartilage, which differed between osteoarthritis and controls.

- This suggests that trafficking of dead or alive gut microbiota physiologically occurs between gut and epiphysial bone marrow.

How might this impact on clinical practice?

- Physiologic subchondral microbiota could enhance cartilage healing and transform components of deep cartilage matrix in metabolites with immunosuppressive properties.

Conversely, dysbiosis of the subchondral microbiota might contribute to some osteoarthritis and account for associations between obesity and osteoarthritis of upper limbs.

in gut microbiota, whereas meta-analysis of the human gut microbiome from urbanised and preagricultural populations showed that the urbanisation/industrialisation process which occurred in recent human history has shaped the gut microbiota through the acquisition and/or loss of specific gut microbes. ${ }^{2}$ Whether some of those gut bacteria could be more toxic for cartilages is not yet proven, but some experimental evidence support a contribution of microbiota to OA. ${ }^{3}$ For instance, OA induced by destabilisation of the medial meniscus is reduced in germ-free mice. ${ }^{4}$ Cartilage damage (induced through cyclic compressive loading) was similarly reduced in mice also submitted to chronic antibiotics to prevent metabolic syndrome, which suggests that the gut microbiome may influence cartilage pathology. ${ }^{5}$ In humans, significant associations were found between individual gut microbiome taxonomies and knee and hip OA Western Ontario and McMaster Universities Osteoarthritis Index pain scores. ${ }^{6}$ Moreover, an association has been found between 
OA and obesity, which occurs even in non-weight-bearing joints in the upper extremities. ${ }^{78}$ Several hypotheses have been put forward to explain those findings: (1) dysbiosis is associated with metabolic syndrome, which could foster OA by itself, ${ }^{9}$ since increased $\mathrm{OA}$ in diet-induced obese animals is associated with greater body fat, but not body mass ${ }^{10}$; (2) in mice, obesity is associated with loss of beneficial Bifidobacteria in gut, while key proinflammatory species gain in abundance, leading to macrophage migration to the synovium and accelerated knee $\mathrm{OA}^{11}$; (3) obesity is associated with compromised gut mucosa, translocation of microbiota and raised serum lipopolysaccharides. ${ }^{7}$ Low-grade inflammation induced by this translocation can prime the proinflammatory innate immune response in joints via toll-like receptor-4 (TLR-4) ${ }^{12}$ and systemic and synovial concentrations of bacterial lipopolysaccharides are positively correlated with both joint inflammatory response and severity of joint degradation (osteophyte score, joint space narrowing). ${ }^{12}$

A more unexpected explanation could be that a similar translocation of gut microbiota occurs in subchondral bone marrow and/or deeper zone of cartilage, which could directly contribute to OA changes. It may do so either by disturbing cartilage metabolism (intestinal microbiota is involved in host physiology through its metabolic products and some metabolites might be toxic for cartilages), or by progressively inducing low-grade inflammation and/or epigenetic changes in chondrocytes and subchondral bone marrow cells. ${ }^{13}$ Such transient translocation of gut microbiota to subchondral bone marrow might also contribute to explain some OA flares.

The present article is a narrative review aiming to gather arguments or evidence from recent literature supporting the hypothesis that translocation of dead or alive commensal bacteria from gut microbiota next to cartilage might contribute to the pathogenesis of some animal and human OA. For this purpose, articles indexed on Medline before June 2019 and dealing with this topic were retrieved using the following keywords crossed together: 'osteoarthritis' or 'cartilage' or 'chondrocytes' AND 'microbiota' or 'dysbiosis' or 'bacteria' or 'commensal' or 'translocation' or 'trafficking' or 'migration' or 'blood' or 'vascular channels'.

\section{THE SILENT TRAFFICKING OF MICROBIOTA FROM GUT TO BLOOD CONTRIBUTES TO THE ONSET OF OTHER RHEUMATIC CONDITIONS}

The main source of leakage of microbiota or their metabolites into the venous or lymphatic circulation is the gut. Such intermittent leakage can be sufficient to trigger the onset and flares of diseases previously considered as only autoimmune. ${ }^{14}$ For instance, Enterococcus gallinarum can migrate in the liver and induce lupus-like disorders associated with hepatitis, either in mice or humans. ${ }^{15}$ Similarly, in a TLR-2-deficiency mouse model, a downregulation of expression of tight junction-associated protein
ZO-1 leads to increased gut permeability and bacterial translocation from gut to liver, promoting autoimmune cholangitis. ${ }^{16}$ Other disorders previously classified as autoimmune might also partly result from over-responses to another kind of self, that is, bacteria from microbiota, which could be seen like family dogs protecting their host from 'wolves' (more virulent bacteria). It might be stressed that gut permeability (loss of integrity between the cells of the small intestine, usually assessed/defined by either the level of serum zonulin, a dual sugar test or the level of lipopolysaccharide found within the blood $)^{17}$ is not synonymous to bacterial translocation. Indeed, many situations with increased gut permeability are not associated with bacterial translocation, which can conversely occur in patients without gut permeability. For instance, most live gut commensal bacteria translocate through the gut epithelial layer using physiologic means. ${ }^{18}$ They are physiologically taken in by $\mathrm{M}$ cells to Peyer's patches and carried by dendritic cells to mesenteric lymph nodes (MLN) to mount IgA responses (which further increases their uptake and growth within gut-associated lymphoid tissue where they promote tolerance) ${ }^{18}$ Further dissemination of those commensals to remote tissues seems to depend mainly on the immune responses in MLNs, which could be much more important than mucosal integrity. This could explain why some conditions recently suspected to be driven by excessive translocation of commensal bacteria from gut to tissues are not associated with overt colitis, whereas trafficking of commensal bacteria towards remote tissues could rather be impaired in conditions like Crohn's disease, due to the very strong immune responses in MLNs.

\section{TRAFFICKING OF DNA MICROBIOTA TO THE JOINTS IS THE RULE, NOT THE EXCEPTION}

In a recent study, Porphyromonas and Bacteroides DNA could be detected in all 183 samples of either rheumatoid arthritis (RA) or OA synovium or synovial fluid. ${ }^{19}$ DNAs of some of those microbiotal bacteria were more abundant in RA synovial tissues, whereas others were more abundant in OA synovial tissues, and they differed in each condition from bacterial DNA found in the corresponding synovial fluids. ${ }^{19}$ It is still unclear whether these nucleic acids originated from immune cells (eg, macrophages) trafficking from mucosae, or from bacteria still living when reaching joints.

\section{BLOOD OF HEALTHY HUMANS IS NOT PERFECTLY STERILE}

Using a 16S targeted metagenomics sequencing pipeline on whole blood from 30 healthy blood donors, it was recently observed that a diversified microbiome exists in healthy blood. ${ }^{20}$ At the phylum level, blood fractions contained mostly bacterial DNA from the Proteobacteria phylum (more than 80\%), but also from Actinobacteria, Firmicutes, and Bacteroidetes. Most of blood bacterial DNA was located in the buffy coat $(93.74 \%)$, but some were included in red blood cells, which contain much 
more bacterial DNA $(6.23 \%)$ than the plasma $(0.03 \%) .{ }^{20}$ Those findings confirm previous observations made using transmission electronic microscopy. ${ }^{21}$ Some of those bacteria could be dead, but others might only be dormant. Indeed, some authors recently claimed that this blood microbiota (bacterial and fungal) could be resuscitated $^{22}$ by using high concentrations of vitamin $\mathrm{K}$ and maintaining bacteria at $43^{\circ} \mathrm{C}$ for at least 72 hours. Of the 28 tested blood samples of healthy individuals, all cultures were positive after 30 days, as also confirmed by Gram staining and transmission electronic microscopy, the bacterial orders being predominantly of phylum Proteobacteria. However, this microbiota is probably prone to remain silent, since in subcultures this blood microbiota reverted to a dormant state. ${ }^{22}$ Like in mucosae, one of the physiological backgrounds of this blood commensal microbiota might be to protect the host from more virulent bacteria in blood and/or endothelial cells. Although this transient resuscitation has not been reproduced yet, the possibility that live bacteria from gut microbiota could reach cartilage deserves some attention.

\section{GUT MICROBIOME WAS RECENTLY FOUND IN HUMAN CARTILAGE}

Using sterile procedure, cartilage was obtained from primary OA knee and hip replacements, and in each location both OA-eroded and preserved cartilages were gathered. ${ }^{23}$ Cartilage of patients free from OA was obtained from 30 fresh cadavers of similar sexes, age and body mass index (BMI). For all samples, 16s RNA gene deep sequencing was done on an Illumina HiSeq. Group OTU differences were confirmed with clade-specific qPCR in a separate confirmation cohort (10 eroded, 10 intact, 10 control samples). In group analysis, there were 63 clade differences between OA and controls. OA was dominated by Betaproteobacteria, whereas controls were characterised by Actinobacteria and Clostridia. A shift was noted in OA towards Gram-negative constituent DNA. A significant difference was also noticed between hip OA and knee OA. Hip OA microbiota was dominated by the phylum Proteobacteria, whereas knee OA microbiota was characterised by Actinobacteria. Similarly, 46 differences were seen in knee controls versus hip controls. ${ }^{23}$ A study in OA shoulder concluded to the opposite since after stringent removal of contamination, only genomic DNA from various Acinetobacter spp and from the Oxalobacteraceae family could be identified in $74 \%$ of rotator cuff tendon tissue samples, but not in humeral head cartilage. ${ }^{24}$ Although those findings also require confirmation, the predominance of Proteobacteria (at least in hip) would be in line with the observation made when studying blood microbiome. ${ }^{22}$ The predominance of Betaproteobacteria in OA cartilage would also fit with the observations that increased abundance of the phylum Proteobacteria is a marker for an unstable gut microbial community observed in patients with metabolic disorders. ${ }^{25}$
HOW MICROBIOTA DNA OR GUT BACTERIA COULD REACH THE CARTILAGE

It has not yet been studied whether this DNA was stored within the subchondral bone marrow, deep cartilage vessels, cartilage matrix or chondrocytes themselves. ${ }^{23}$ How this DNA (and perhaps still living bacteria from microbiota) reaches cartilage remains unclear. Unlike most tissues, articular cartilage does not have blood vessels, nerves or lymphatics in the superficial, middle and deep zones, so that most of the nutrition of the articular cartilage occurs by diffusion from the synovial fluid and subchondral bone marrow. The cartilage matrix restricts materials by size, charge and molecular configuration. ${ }^{26}$ It is estimated that the average pore size within the cartilage extracellular matrix is approximately $6.0 \mathrm{~nm},{ }^{27}$ whereas the size of most bacteria ranges from 100 to $4000 \mathrm{~nm}$, and the size of bacterial spores is around $100 \mathrm{~nm}$. Therefore, penetration of bacteria through the superficial zone of cartilage does not seem possible, unless large erosions occur. The same could hold true for bacterial DNA, for another reason: whereas the charge of DNA is negative, only the positive charge of a substance has the potential to promote its accumulation inside the cartilage matrix, since even neutral molecules do not diffuse in cartilage. ${ }^{28}$ This applies to most bacteria, since both Gram-positive and Gram-negative bacterial cell walls have a negative charge.

Conversely, calcified cartilage is permeable to small solutes, and the subchondral circulation may make a significant contribution to the nutrition of deep cartilage. ${ }^{29}$ Within the calcified zone of core areas, voluminous pieces of permanent cartilage are metabolically supplied via vascular channels (figure 1 ) ${ }^{30}$ Moreover, the osteochondral junction displays neoangiogenesis in case of OA. ${ }^{31}$ Interestingly, bacteria can bind to growth cartilage and disrupt the blood supply in pigs and chickens. Bacteria were also observed within cartilage canals from young horses (foals) with septic arthritis/osteomyelitis, and canals acutely or chronically infected were associated with ischaemic chondronecrosis in the articular-epiphysial cartilage complex. ${ }^{32}$

Pathologic replication of microbiota could similarly contribute to some growth cartilage defects: in chicken embryo, exposure to microbiota-derived lipopolysaccharide inhibits chondrogenesis and retards chondrocyte hypertrophy in the growth plate through elevating Sox 9 expression. ${ }^{33}$

As vascular channels persist in adults ${ }^{32}$ (including in some areas like nose, thyroid cartilage and rib cartilages, targets of relapsing polychondritis, where cartilage cannot be supplied by synovial nutriments), they may facilitate the trafficking of blood microbiota in those locations and subchondral bone marrow. Transient presence of non-pathogenic bacteria within adult vascular channels has not been reported so far in humans, but bacteria from gut microbiota may persist in a latent state within subchondral bone marrow close to the deeper zone of cartilage, since they could find there the same anaerobic 
Cartilage

Osteoarthritis

microcracks and deep fissures

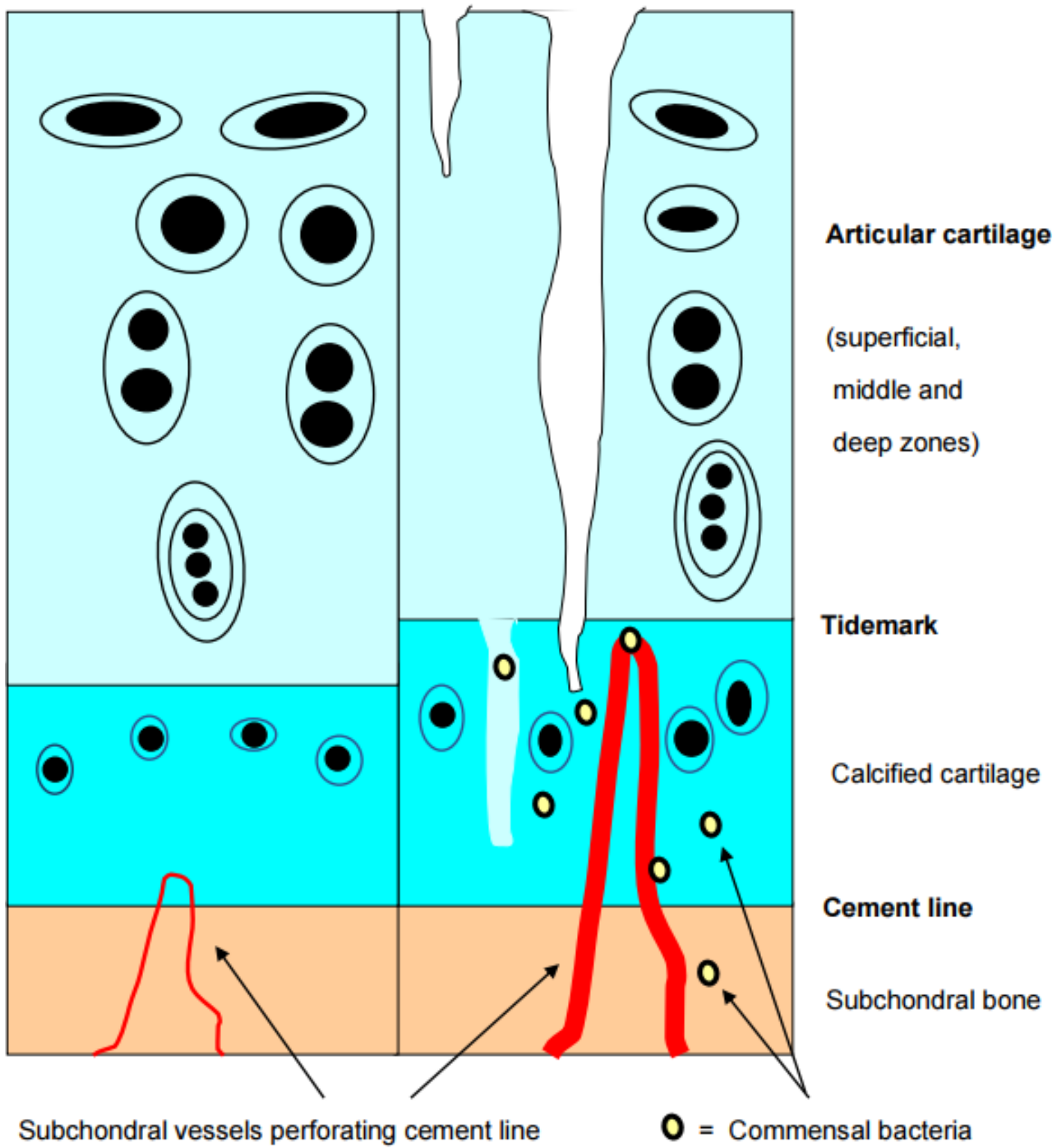

\section{and getting close to tidemark in osteoarthritis}

Figure 1 Within the calcified zone of core areas, voluminous pieces of permanent cartilage are metabolically supplied via vascular channels. Those channels are more numerous and enlarged in osteoarthritis, and might allow commensal bacteria to migrate from blood into calcified cartilage and subchondral bone, and even in articular cartilage, through cracks in the tidemark.

metabolism ${ }^{24}$ as in the gut. Such transient invasion might occur even before birth, as Proteobacteria, the leading subsets of gut microbiota found in healthy blood and cartilage, are part of a normal, healthy, placental microbiome. ${ }^{34}$ Another reason to search for gut microbiota in human epiphysial bone marrow is the demonstration of the influence of commensal microbiota on human bone metabolism (antianabolic and procatabolic effects), which appears mediated in mice via local disruption of insulin-like growth factor-1 signalling. ${ }^{35}$

\section{CAN BACTERIA INFECT SOME CHONDROCYTES?}

Some studies even addressed the possibility of bacterial infection of chondrocytes: Mycoplasma synoviae, a major poultry pathogen, could invade primary chicken 
chondrocytes in vitro. ${ }^{36}$ M. synoviae upregulated gene expression in those chondrocytes, including the expression of the gene-encoding peptidylarginine deiminase type III, which is responsible for the post-translational citrullination of proteins. ${ }^{37}$

In humans, Porphyromonas gingivalis can adhere and infect primary human chondrocytes in vitro, ${ }^{38}$ which increases apoptosis, partly through the phosphoglycerol dihydroceramide lipids of the bacteria. Some strains of Streptococcus pyogenes could also invade chondrocytic cell lines, leading to strong expression of matrix metalloproteinase-13 and degradation of the extracellular matrix. ${ }^{39}$

It must however be stressed that in vivo such infections remain still quite hypothetic, although an acute borrelial arthritis was observed following autologous chondrocyte transplantation for repair of a cartilage defect in a patient who had 15 years earlier an expanding erythematous lesion after a tick bite. Borrelial DNA sequences were detected in the synovial fluid of the grafted joint through PCR, and the diagnosis was confirmed by culture of Borrelia burgdorferi, suggesting that the bacteria had remained dormant in cells of the grafted cartilage for 15 years. ${ }^{40}$

\section{COULD GUT MICROBIOTA HAVE A PHYSIOLOGICAL ROLE IN NORMAL SUBCHONDRAL BONE MARROW?}

The finding of gut microbiota in all samples of human cartilage studied by Dunn et al, ${ }^{23}$ which differed according to joints, would imply that the presence of those bacteria in cartilage is not random, and could play a physiological role, despite their very low numbers and steady state. For instance, their ability to transform some components of deep cartilage matrix in metabolites with immunosuppressive properties might be one reason for their unexpected presence close to cartilage. It was indeed recently shown that chondroitin sulfate disaccharides (which inhibit the growth of Proteobacteria) were likely butyrogenic substrates, and when added to mice diet, increased total short-chain fatty acids (SCFA), as well as butyrate levels, under both healthy and stressed conditions. ${ }^{25}$ Similarly, keratan sulfate from shark cartilage also had a strong effect on gut microbiota in mice, and induced a drastic increase in the abundance of Lactobacillus spp (16-fold in female mice, and twofold in male mice), which is an important group of SCFA producers. ${ }^{41}$ Although yet only observed in gut, the hypothesis that production of SCFA by microbiota embedded in bone marrow vessels close to the deeper zone of hyaline epiphysial cartilage (and thus exposed to appropriate substrates like chondroitin sulfate or keratin sulfate) could similarly sustain a tolerogenic niche to protect haematopoietic and stromal bone marrow stem cells from excessive immune responses might make sense. ${ }^{10}$ Another rationale for the presence of microbiota at the edge of cartilage, close to bone marrow niches, might be the intrinsic property of ectopic gut microbiota to contain the expansion of pathogenic bacteria. Indeed those 'self-bacteria' might act as mercenaries to avoid growing of more dangerous species in tissues where excessive adaptive immune responses are not welcome. A last possibility could be the potential of some bacteria from gut microbiota to enhance cartilage healing. Indeed, MRL/MpJ mice (which are substantially protected from developing post-traumatic $\mathrm{OA}$, a trait with strong correlation to the ability to heal ear wounds) have substantial differences in gut microbiota composition compared with non-healer mice. ${ }^{42}$ Moreover, the cartilage healing trait is partially transferable to non-healer B6 mice via a gut microbiota transplant, and is associated with shifts in some gut microbiotic taxa. ${ }^{42}$

\section{HOW GUT BACTERIA COULD AFFECT CARTILAGE IN OA}

The osteochondral plate angiogenesis occurring early in $\mathrm{OA}^{31}$ could facilitate transient migration of still living bacteria or some of their products, up to the deeper layers of cartilage. In a study on 431 patients with knee OA, baseline lipopolysaccharide-binding protein (LBP) and time-integrated concentration of LBP were associated with worsening joint space width and narrowing, even after adjusting for treatment group, age, body mass index and baseline radiographic measures. ${ }^{43}$ Soluble TLR-4 levels were also associated with knee OA progression over 16-18 months. ${ }^{43}$ Excess of microbiotal antigens in the deeper layers of cartilage might foster low-grade inflammation and cartilage degradation, in the same way as pathogenic bacterial antigens, like borrelia ${ }^{44}$ tightly and long-lastingly bound to human cartilage via integrin alpha3beta1, can stimulate the production of proinflammatory mediators in primary human chondrocytes. ${ }^{45}$ Focal dysbiosis induced by migration of pathogenic bacteria of microbiota in subchondral bone marrow could also affect the epigenetic of chondrocytes, since microbial metabolites can induce epigenetic modifications (ie, changes in DNA methylation and micro-RNA expression) of some human cells, as shown in obese patients. ${ }^{46}$

\section{THE DIFFERENCES OF MICROBIOTA OBSERVED BETWEEN HIP AND KNEE CARTILAGE, EITHER IN PATIENTS WITH OA OR CONTROLS, MIGHT BE IN LINE WITH DIFFERENCES IN CHONDROCYTE METABOLISMS AND DNA METHYLATION ACCORDING TO JOINTS}

Hip and knee cartilage are not identical: genome-wide DNA methylation was measured in 31 preserved and lesioned cartilage sample pairs (14 knees and 17 hips) from patients who underwent a total joint replacement due to primary OA. Irrespective of OA pathophysiology, a total of $6272 \mathrm{CpG}$ dinucleotides were differentially methylated between both joints. ${ }^{47}$ The consistent gene expression differences between knee and hip articular cartilage in differentially methylated regions marked transcriptomic differences among HOX genes. ${ }^{47}$ Major differences in methylation profiles of hip and knee chondrocytes were confirmed in another study, which also concluded that either hip or knee OA clustered into two 


\section{Box 1 Research agenda: bullet points to address}

- Are the findings of normal gut microbiota in human cartilage, differing between osteoarthritis $(\mathrm{OA})$ and controls, as well as between hip and knee, reproducible?

- Does the nature of joint shape the profile of cartilage microbiota more than the blood and gut microbiome?

- Do glycans from cartilage shape the composition of the cartilage microbiota in the same way as glycans from diet shape the gut microbiota?

- Are changes in gut microbiota according to age, body mass index (BMI), diet, exercise, sex and/or hormonal background, associated with simultaneous changes in the cartilage microbiota?

Do differences in cartilage microbiota in some ethnic groups contribute to explain the findings of epidemiologic studies of $0 A$ across races (ie, lower frequency of $O A$ in black and Asians)?

groups. ${ }^{48}$ Simultaneous analysis of chondrocytes DNA methylation and microbiota composition in hip and knee normal and OA cartilages will be necessary to assess whether changes in microbiota correlate with clusters of differently methylated genes in hip versus knee cartilage, and different metabolisms according to joints.

\section{CONCLUSION}

Until recently, most students were taught to consider only bacteria growing in cultures and deserving antibiotics. Consequently, many physicians still turn a blind eye on microbiota, felt as either neglectable harmless bacteria in the mucosae, or contamination when found in other tissues. The poor clinical efficiency of doxycycline in OA of the knee and hip despite significant slowing of joint space narrowing $^{49}$ also reinforced the disinterest for the involvement of bacteria in the pathogenesis of OA and other cartilage disorders. In fact, antibiotics can worsen dysbiosis rather than improve it,${ }^{50}$ and the confirmation of the presence of gut microbiota close to or within the normal deeper layers of cartilage ${ }^{23}$ would be a revolution in the understanding of at least some subsets of OA (including those associated with metabolic syndrome). It would also offer new therapeutic avenues, at least for better preventing them. As OA is more frequent in older women, it will be interesting to seek for differences, both in faeces and in cartilage, according to sex and age. Indeed, although the effect of sex on the gut microbiota appears to be less influential than that of other factors, most animal and human studies have shown sex-related differences in gut microbiota. ${ }^{51}$ Age also induces changes in gut microbiota: a cross-sectional study of faeces of 367 healthy Japanese subjects (0-104 years) using hierarchical Ward's linkage clustering of genera indicated some patterns and transition points in the compositional changes in gut microbiota with age: five clusters were identified, with median (IQR) ages of 3 (0-35), 33 (24-45), 42 (32-62), 77 (36-84) and 94 (86-98) years. ${ }^{52}$ Focusing on the incidence exercise and diet on joint/cartilage microbiota should also be instructive. Indeed, exercise ${ }^{5354}$ and $\operatorname{diet}^{55} 56$ modify gut microbiota in humans and murine models, and this may translate into differences within the joints and cartilages, too. Further studies of cartilage microbiome and microbiota across different ethnic groups, BMI, joints, age, exercise, diet and even glycan composition (box 1) are therefore eagerly expected, first to confirm, then to extend the preliminary finding of gut microbiome in human cartilage, which seems to differ in controls and OA. ${ }^{23}$

Contributors JMB wrote the first draft of this review, which was extensively reorganised and improved by the other authors.

Funding The authors have not declared a specific grant for this research from any funding agency in the public, commercial or not-for-profit sectors.

Competing interests None declared.

Patient consent for publication Not required.

Provenance and peer review Not commissioned; externally peer reviewed.

Data availability statement Data is avaible in a public, open access repository.

Open access This is an open access article distributed in accordance with the Creative Commons Attribution Non Commercial (CC BY-NC 4.0) license, which permits others to distribute, remix, adapt, build upon this work non-commercially, and license their derivative works on different terms, provided the original work is properly cited, appropriate credit is given, any changes made indicated, and the use is non-commercial. See: http://creativecommons.org/licenses/by-nc/4.0/.

\section{REFERENCES}

1. Berenbaum F, Wallace IJ, Lieberman DE, et al. Modern-day environmental factors in the pathogenesis of osteoarthritis. Nat Rev Rheumatol 2018;14:674-81.

2. Mancabelli L, Milani C, Lugli GA, et al. Meta-analysis of the human gut microbiome from urbanized and pre-agricultural populations. Environ Microbiol 2017;19:1379-90.

3. Szychlinska MA, Di Rosa M, Castorina A, et al. A correlation between intestinal microbiota dysbiosis and osteoarthritis. Heliyon 2019;5:e01134.

4. Ulici V, Kelley KL, Azcarate-Peril MA, et al. Osteoarthritis induced by destabilization of the medial meniscus is reduced in germ-free mice. Osteoarthritis Cartilage 2018;26:1098-109.

5. Guss JD, Ziemian SN, Luna M, et al. The effects of metabolic syndrome, obesity, and the gut microbiome on load-induced osteoarthritis. Osteoarthritis Cartilage 2019;27:129-39.

6. Boer CG, Radjabzadeh D, Uitterlinden AG, et al. The role of the gut microbiome in osteoarthritis and joint pain [Abstract]. Osteoarthritis Cartilage 2017;25.

7. Metcalfe D, Harte AL, Aletrari MO, et al. Does endotoxaemia contribute to osteoarthritis in obese patients? Clin Sci 2012;123:627-34.

8. Sellam J, Berenbaum F. Is osteoarthritis a metabolic disease? Joint Bone Spine 2013;80:568-73.

9. Courties A, Sellam J, Berenbaum F. Metabolic syndrome-associated osteoarthritis. Curr Opin Rheumatol 2017;29:214-22.

10. Collins $\mathrm{KH}$, Paul HA, Reimer RA, et al. Relationship between inflammation, the gut microbiota, and metabolic osteoarthritis development: studies in a rat model. Osteoarthritis Cartilage 2015;23:1989-98.

11. Schott EM, Farnsworth CW, Grier A, et al. Targeting the gut microbiome to treat the osteoarthritis of obesity. JCl Insight 2018;3.

12. Huang ZY, Stabler T, Pei FX, et al. Both systemic and local lipopolysaccharide (LPS) burden are associated with knee OA severity and inflammation. Osteoarthritis Cartilage 2016;24:1769-75.

13. Jeffries MA, Donica M, Baker LW, et al. Genome-Wide DNA methylation study identifies significant epigenomic changes in osteoarthritic Subchondral bone and similarity to overlying cartilage. Arthritis Rheumatol 2016;68:1403-14.

14. Mu Q, Kirby J, Reilly CM, et al. Leaky gut as a danger signal for autoimmune diseases. Front Immunol 2017;8:598.

15. Manfredo Vieira S, Hiltensperger M, Kumar V, et al. Translocation of a gut pathobiont drives autoimmunity in mice and humans. Science 2018;359:1156-61.

16. Ma H-D, Zhao Z-B, Ma W-T, et al. Gut microbiota translocation promotes autoimmune cholangitis. J Autoimmun 2018;95:47-57. 
17. Leech B, Mclntyre E, Steel A, et al. Risk factors associated with intestinal permeability in an adult population: a systematic review. Int $J$ Clin Pract 2019:e13385.

18. Fine RL, Manfredo Vieira S, Gilmore MS, et al. Mechanisms and consequences of gut commensal translocation in chronic diseases. Gut Microbes 2019;62:1-14.

19. Zhao Y, Chen B, Li S, et al. Detection and characterization of bacterial nucleic acids in culture-negative synovial tissue and fluid samples from rheumatoid arthritis or osteoarthritis patients. Sci Rep 2018;8:14305.

20. Païssé S, Valle C, Servant F, et al. Comprehensive description of blood microbiome from healthy donors assessed by 16S targeted metagenomic sequencing. Transfusion 2016;56:1138-47.

21. Potgieter M, Bester J, Kell DB, et al. The dormant blood microbiome in chronic, inflammatory diseases. FEMS Microbiol Rev 2015;39:567-91.

22. Panaiotov S, Filevski G, Equestre M, et al. Cultural isolation and characteristics of the blood microbiome of healthy individuals. Adv Microbiol 2018;08:406-21.

23. Dunn C, Velasco C, Rivas A, et al. Identification of a human cartilage microbial DNA signature and characterization of distinct microbiome profiles associated with osteoarthritis [abstract 2005]. Arthritis Rheumatol 2018;70(suppl 10).

24. Quu B, Al K, Pena-Diaz AM, et al. Cutibacterium acnes and the shoulder microbiome. J Shoulder Elbow Surg 2018;27:1734-9.

25. Liu F, Zhang N, Li Z, et al. Chondroitin sulfate disaccharides modified the structure and function of the murine gut microbiome under healthy and stressed conditions. Sci Rep 2017;7:6783.

26. Sophia Fox AJ, Bedi A, Rodeo SA. The basic science of articular cartilage: structure, composition, and function. Sports Health 2009:1:461-8

27. Mow VC, Ratcliffe A, Poole AR. Cartilage and diarthrodial joints as paradigms for hierarchical materials and structures. Biomaterials 1992:13:67-97.

28. Sterner B, Harms M, Wöll S, et al. The effect of polymer size and charge of molecules on permeation through synovial membrane and accumulation in hyaline articular cartilage. Eur J Pharm Biopharm 2016;101:126-36.

29. Arkill KP, Winlove CP. Solute transport in the deep and calcified zones of articular cartilage. Osteoarthritis Cartilage 2008;16:708-14.

30. Gabner S, Häusler G, Böck P. Vascular canals in permanent hyaline cartilage: development, corrosion of nonmineralized cartilage matrix, and removal of matrix degradation products. Anat Rec 2017;300:1067-82.

31. Pesesse L, Sanchez C, Henrotin Y. Osteochondral plate angiogenesis: a new treatment target in osteoarthritis. Joint Bone Spine 2011;78:144-9.

32. Wormstrand B, Østevik L, Ekman S, et al. Septic Arthritis/ Osteomyelitis may lead to Osteochondrosis-Like lesions in foals. Vet Pathol 2018;55:693-702.

33. Cheng X, Li P-Z, Wang G, et al. Microbiota-derived lipopolysaccharide retards chondrocyte hypertrophy in the growth plate through elevating SOX9 expression. J Cell Physiol 2019;234:2593-605.

34. Mor G, Kwon J-Y. Trophoblast-microbiome interaction: a new paradigm on immune regulation. Am J Obstet Gynecol 2015;213(4 Suppl):S131-S137.

35. Novince CM, Whittow CR, Aartun JD, et al. Commensal gut microbiota immunomodulatory actions in bone marrow and liver have catabolic effects on skeletal homeostasis in health. Sci Rep 2017;7:5747.

36. Dusanić D, Berčič RL, Cizelj I, et al. Mycoplasma synoviae invades non-phagocytic chicken cells in vitro. Vet Microbiol 2009;138:114-9.
37. Cizelj I, Dušanić D, Benčina D, et al. Mycoplasma and host interaction: In vitro gene expression modulation in Mycoplasma synoviae and infected chicken chondrocytes. Acta Vet Hung 2016;64:26-37.

38. Pischon N, Röhner E, Hocke A, et al. Effects of Porphyromonas gingivalis on cell cycle progression and apoptosis of primary human chondrocytes. Ann Rheum Dis 2009;68:1902-7.

39. Sakurai A, Okahashi N, Maruyama F, et al. Streptococcus pyogenes degrades extracellular matrix in chondrocytes via MMP-13. Biochem Biophys Res Commun 2008;373:450-4.

40. Marlovits S, Khanakah G, Striessnig G, et al. Emergence of Lyme arthritis after autologous chondrocyte transplantation. Arthritis Rheum 2004;50:259-64.

41. Shang $Q$, Li Q, Zhang $M$, et al. Dietary keratan sulfate from shark cartilage modulates gut microbiota and increases the abundance of Lactobacillus spp. Mar Drugs 2016;14:224.

42. Dunn C, Velasco C, Andrews M, et al. Murine ear would cartilage superhealer trait is associated with gut microbiota changes and is transferable to non-healer mice by gut microbiome transplant [abstract]. Arthritis Rheumatol 2018;70(suppl 10).

43. Huang ZY, Perry E, Huebner JL, et al. Biomarkers of inflammation LBP and TLR- predict progression of knee osteoarthritis in the DOXY clinical trial. Osteoarthritis Cartilage 2018;26:1658-65.

44. Bockenstedt LK, Gonzalez DG, Haberman AM, et al. Spirochete antigens persist near cartilage after murine Lyme borreliosis therapy. J Clin Invest 2012;122:2652-60.

45. Behera AK, Durand E, Cugini C, et al. Borrelia burgdorferi BBB07 interaction with integrin alpha3beta1 stimulates production of pro-inflammatory mediators in primary human chondrocytes. Cell Microbiol 2008;10:320-31.

46. Cuevas-Sierra A, Ramos-Lopez O, Riezu-Boj Jl, et al. Diet, gut microbiota, and obesity: links with host genetics and epigenetics and potential applications. Adv Nutr 2019;10(suppl 1):S17-S30.

47. den Hollander W, Ramos YFM, Bos SD, et al. Knee and hip articular cartilage have distinct epigenomic landscapes: implications for future cartilage regeneration approaches. Ann Rheum Dis 2014;73:2208-12.

48. Rushton MD, Reynard LN, Barter MJ, et al. Characterization of the cartilage DNA methylome in knee and hip osteoarthritis. Arthritis Rheumatol 2014;66:2450-60.

49. Nüesch E, Rutjes AW, Trelle S, et al. Doxycycline for osteoarthritis of the knee or hip. Cochrane Database Syst Rev 2009.

50. Yoon MY, Yoon SS. Disruption of the gut ecosystem by antibiotics. Yonsei Med J 2018;59:4-12.

51. Kim YS, Unno T, Kim B-Y, et al. Sex differences in gut microbiota. World J Mens Health 2019;37.

52. Odamaki T, Kato K, Sugahara $\mathrm{H}$, et al. Age-related changes in gut microbiota composition from newborn to centenarian: a crosssectional study. BMC Microbiol 2016;16:90.

53. Morita E, Yokoyama $\mathrm{H}$, Imai $\mathrm{D}$, et al. Aerobic exercise training with Brisk walking increases intestinal Bacteroides in healthy elderly women. Nutrients 2019;11:E868.

54. Mailing LJ, Allen JM, Buford TW, et al. Exercise and the gut microbiome: a review of the evidence, potential mechanisms, and implications for human health. Exerc Sport Sci Rev 2019;47:75-85.

55. Li Y, Luo W, Deng Z, et al. Diet-Intestinal microbiota axis in osteoarthritis: a possible role. Mediators Inflamm 2016;2016:3495173.

56. Rios JL, Bomhof MR, Reimer RA, et al. Protective effect of prebiotic and exercise intervention on knee health in a rat model of dietinduced obesity. Sci Rep 2019;9:3893. 